\title{
Homographic Solutions of the N-body Generalized Lennard-Jones System
}

\author{
Bowen $\mathrm{Liu}^{1 *}$ \\ ${ }^{1}$ Chern Institute of Mathematics \\ Nankai University, Tianjin 300071, China
}

June 4, 2018

\begin{abstract}
In this paper, we obtain the existence of non-planar circular homographic solutions and noncircular homographic solutions of the $(2+N)$ - and $(3+N)$-body problems of the Lennard-Jones system. These results show the essential difference between the Lennard-Jones potential and the Newton's potential of universal gravitation.
\end{abstract}

2010 MS classification: $34 \mathrm{C} 25,70 \mathrm{H} 12,82 \mathrm{C} 22$

Key words: Homographic solution, Lennard-Jones potential, non-planar circular solution.

Running title: Homographic Solutions of N-body Generalized Lennard-Jones System.

\section{Introduction}

In this paper, we consider the existence of special homographic solutions of the generalized Lennard-Jones (L-J for short) system. The configuration space in $\mathbf{R}^{3}$ is defined by

$$
X=\left\{q=\left(q_{1}, q_{2}, \ldots, q_{N}\right) \in\left(\mathbf{R}^{3}\right)^{N} \mid \sum_{k=1}^{N} m_{k} q_{k}=0, q_{k} \neq q_{j}, k \neq j\right\} .
$$

The generalized $N$-body L-J potential is given by

$$
U(q)=\sum_{k<j} m_{k} m_{j}\left(\frac{1}{\left|q_{k}-q_{j}\right|^{\beta}}-\frac{1}{\left|q_{k}-q_{j}\right|^{\alpha}}\right) .
$$

${ }^{*}$ Partially supported by NSFC (No. 11131004, No. 11671215). Email: bowen.liu@mail.nankai.edu.cn 
For simplicity, we consider the case of $m_{i}=m_{j}=1$. By the Newton's second law, the motion equations are given by

$$
\ddot{q}=-\nabla U(q),
$$

where $\nabla U(q)$ is the gradient of $U(q)$. Then the motion of $q_{k}$ satisfies

$$
\ddot{q}_{k}=\sum_{j \neq k}\left(\frac{\beta}{\left|q_{k j}\right|^{\beta+2}}-\frac{\alpha}{\left|q_{k j}\right|^{\alpha+2}}\right)\left(q_{k}-q_{j}\right),
$$

where $q_{k j}=q_{k}-q_{j}$ and $q_{k j}=-q_{j k}$. Let $q=q(t)$ be a solution of (1.2). It is flat, if $q(t)$ is contained in a plane $\Pi(t)$ in $\mathbf{R}^{3}$ for any $t \in \mathbf{R}$. It is planar, if there is a plane $\Pi$ in $\mathbf{R}^{3}$ which contains $q(t)$ for all $t \in \mathbf{R}$.

In the literature, researchers obtained many results of the planar solutions of the $N$-body problems of the L-J potential. In 2004, when $\alpha=6, \beta=12, a=2$ and $b=1$, Corbera, Llibre and Pérez-Chavela in [1] and [2] obtained the existence of the constant solutions, the circular solutions and central configurations of the planar 2- and 3-body problems of L-J system. In [3], Jones studied the central configurations and proved $N$-gon and $(N+1)$-gon are the central configurations of the planar L-J system. In [6], Sbano and Southall proved the existence of the planar periodic solutions of the $N$-body problem of L-J system when period is large enough by the mountain pass theorem. Readers may refers to [4] and [5] for literature review on the Lennard-Jones potential.

In this paper, we focus on the flat solutions and the non-flat solutions. We define that for $1 \leq k \leq N, r \in \mathbf{R}^{+}$and $\omega_{k}=\frac{2 \pi k}{N}$,

$$
Q_{1}=\left(0,0, r_{0}\right), Q_{2}=\left(0,0,-r_{0}\right), Q_{3}=(0,0,0), q_{k}=\phi(\lambda) r_{0}\left(\cos \omega_{k}, \sin \omega_{k}, 0\right),
$$

where $\phi(\lambda)=\sqrt{\lambda^{2}-1}$ and define

$$
E(t)=\left(\begin{array}{ccc}
\cos \omega(t) & -\sin \omega(t) & 0 \\
\sin \omega(t) & \cos \omega(t) & 0 \\
0 & 0 & 1
\end{array}\right)
$$

If $\omega(t)=\omega_{0} t$ for some constant $\omega_{0} \neq 0$, we write $E(t)$ as $E_{\omega_{0}}(t)$ for simplicity.

At first, we consider the circular solution $q(t)$ of the $(2+N)$-body problem satisfying

$$
q(t)=E_{\omega_{0}}(t) q_{0},
$$

where $q_{0}=\left(Q_{1}, Q_{2}, q_{1}, \ldots, q_{N}\right) \in\left(\mathbf{R}^{3}\right)^{2+N}$ as in (1.3) and $E_{\omega_{0}}(t)$ is given by (1.4). Note that angular momentum is a constant vector parallel with the $z$-axis, i.e., $\dot{\omega} \equiv \omega_{0}$. In this case, $q(t)$ is the solution of (1.2) only if there exists a $\lambda$ such that following equation holds.

$$
\operatorname{diag}\left(-\omega_{0}^{2},-\omega_{0}^{2}, 0\right) q_{0}=\nabla U(q)
$$


Theorem 1.1. When $0<\alpha<\beta, N \geq 2$, there exists $\lambda_{1}$ which is given by (iii) of Lemma 2.1 such that for any $\lambda \geq \max \left\{2, \lambda_{1}\right\}, q(t)=E_{\omega_{0}}(t) q_{0} \in\left(\mathbf{R}^{3}\right)^{2+N}$ satisfying (1.5) is a solution of (1.2) where $\omega_{0}$ is defined by (1.4) and satisfies

$$
\omega_{0}= \pm\left(\frac{\theta_{\alpha}}{\left(\sqrt{\lambda^{2}-1} r_{0}\right)^{\alpha+2}}-\frac{\theta_{\beta}}{\left(\sqrt{\lambda^{2}-1} r_{0}\right)^{\beta+2}}+\frac{2 \alpha}{\left(\lambda r_{0}\right)^{\alpha+2}}-\frac{2 \beta}{\left(\lambda r_{0}\right)^{\beta+2}}\right)^{\frac{1}{2}},
$$

$r_{0}=\frac{G_{1}(\lambda)}{\sqrt{\lambda^{2}-1}}, \theta_{\alpha}$ and $\theta_{\beta}$ are constant given by (2.1), and $G_{1}(\lambda)$ is given by

$$
G_{1}(\lambda)=\frac{\sqrt{\lambda^{2}-1}}{2 \lambda}\left(\frac{\beta\left(2 \lambda^{\beta+2}+2^{2+\beta} N\right)}{\alpha\left(2 \lambda^{\alpha+2}+2^{\alpha+2} N\right)}\right)^{\frac{1}{\beta-\alpha}}, \quad \text { for } \quad \lambda \geq 2 .
$$

By adding another body $Q_{3}$ at the center of mass, there exists a class of special homographic solutions of the $(3+N)$-body prolem when $N \geq 2$. Suppose that

$$
q(t)=E_{\omega_{0}}(t) q,
$$

where $q=\left(Q_{1}, Q_{2}, Q_{3}, q_{1}, \ldots, q_{N}\right) \in\left(\mathbf{R}^{3}\right)^{3+N}$ is given by (1.3) and $E_{\omega_{0}}(t)$ is given by (1.4).

Proposition 1.2. When $0<\alpha<\beta$, there exists a $\lambda$ such that for any $\lambda \geq \max \left\{2, \lambda_{2}\right\}, q(t)=$ $E_{\omega_{0}}(t) q$ satisfying (1.9) is the homographic solution of the system (1.2), where $r_{0}=\frac{G_{2}(\lambda)}{\sqrt{\lambda^{2}-1}}, E_{\omega_{0}}(t)$ is defined by (1.4), $\omega_{0}$ satisfying

$$
\omega_{0}= \pm\left(\frac{\theta_{\alpha}+\alpha}{\left(\sqrt{\lambda^{2}-1} r_{0}\right)^{\alpha+2}}-\frac{\theta_{\beta}+\beta}{\left(\sqrt{\lambda^{2}-1} r_{0}\right)^{\beta+2}}+\frac{2 \alpha}{\left(\lambda r_{0}\right)^{\alpha+2}}-\frac{2 \beta}{\left(\lambda r_{0}\right)^{\beta+2}}\right)^{\frac{1}{2}},
$$

and $G_{2}(\lambda)$ is given by

$$
G_{2}(\lambda)=\frac{\sqrt{\lambda^{2}-1}}{2 \lambda}\left(\frac{\beta\left(2^{\beta+2} \lambda^{\beta+2}+2 \lambda^{\beta+2}+2^{\beta+2} N\right)}{\alpha\left(2^{\alpha+2} \lambda^{\alpha+2}+2 \lambda^{\alpha+2}+2^{\alpha+2} N\right)}\right)^{\frac{1}{\beta-\alpha}} .
$$

In Theorem 1.3, we prove the existence of the non-circular homographic solutions. Suppose

$$
q(t)=r(t) E(t) q_{0},
$$

where $q_{0}=\left(Q_{1}, Q_{2}, q_{1}, \ldots, q_{N}\right) \in\left(\mathbf{R}^{3}\right)^{2+N}$ is given by (1.3), $E(t)$ is defined by (1.4) and $r_{0} \equiv 1$. When $r \in \mathbf{R}^{+}$, we define

$$
\Psi(r)=\frac{\beta\left(N 2^{\beta+2}+2 \lambda^{\beta+2}\right)}{(\beta+1)(2 \lambda)^{\beta+2} r^{\beta+1}}-\frac{\alpha\left(N 2^{\alpha+2}+2 \lambda^{\alpha+2}\right)}{(\alpha+1)(2 \lambda)^{\alpha+2} r^{\alpha+1}} .
$$

Define $\bar{r}$ as the only positive root $\Psi^{\prime}(r)=0$ by

$$
\bar{r}=\frac{1}{2 \lambda}\left(\frac{\beta\left(N 2^{\beta+2}+2 \lambda^{\beta+2}\right)}{\alpha\left(N 2^{\alpha+2}+2 \lambda^{\alpha+2}\right)}\right)^{\frac{1}{\beta-\alpha}} .
$$


Theorem 1.3. When $0<\alpha<\beta$ and $N \geq 2$, there exists $\lambda_{0}$ given by Lemma 2.3 such that for $\lambda \geq \lambda_{0}, q(t)=r(t) E(t) q_{0}$ satisfying (1.12) is the homographic solution of (1.2) where $r(t)$ is the solution of following Hamiltonian system with given Hamiltonian energy

$$
\left\{\begin{array}{c}
\ddot{r}=-\nabla \Psi(r)=\frac{N \beta}{\lambda^{\beta+2} r^{\beta+1}}-\frac{N \alpha}{\lambda^{\alpha+2} r^{\alpha+1}}+\frac{2 \beta}{2^{\beta+2} r^{\beta+1}}-\frac{2 \alpha}{2^{\alpha+2} r^{\alpha+1}}, \\
H(r, \dot{r} ; a, b)=h, \\
r(\tau)=r(0), \quad \dot{r}(\tau)=\dot{r}(0),
\end{array}\right.
$$

where $h$ satisfies $\Psi(\bar{r})<h<\Psi(\Lambda), \omega(t)$ is defined by $E(t)$ and satisfying

$$
\omega(t)= \pm \int_{0}^{t} \sqrt{\frac{(N-2) \beta}{(\lambda r)^{\beta+2}}-\frac{(N-2) \alpha}{(\lambda r)^{\alpha+2}}+\frac{2 \beta}{(2 r)^{\beta+2}}-\frac{2 \alpha}{(2 r)^{\alpha+2}}-\frac{\theta_{\beta}}{(\phi r)^{\beta+2}}+\frac{\theta_{\alpha}}{(\phi r)^{\alpha+2}}} \mathrm{~d} t
$$

$\Lambda$ is given by

$$
\Lambda \equiv \frac{1}{2 \lambda \phi}\left(\frac{\beta\left((N-2)(2 \phi)^{\beta+2}+2(\lambda \phi)^{\beta+2}-\theta_{\beta}(2 \lambda)^{\beta+2}\right)}{\alpha\left((N-2)(2 \phi)^{\alpha+2}+2(\lambda \phi)^{\alpha+2}-\theta_{\alpha}(2 \lambda)^{\alpha+2}\right)}\right)^{\frac{1}{\beta-\alpha}} .
$$

It is well known that in the celestial mechanics, if the configuration is flat, the solution must be planar, i.e., the motion must be in a given fixed plane; if the configuration is 3-dimensional, the corresponding solution must be homothetic. In Theorem 1.1 and Theorem 1.3 , if $N=2$, the homographic solution is flat but it is not planar; if $N \geq 3$, the configuration is 3-dimensional, and the corresponding solution is circular but not homothetic. Theses results show the essiential differences between the Newton's law of universal gravitation and the L-J potential.

\section{The Homographic Solutions}

\subsection{The circular homographic solutions of the $(2+N)$-and $(3+N)$-body cases}

In this subsection, we consider (1.5). The shape of configuration $q$ is uniquely determined by $\lambda$. We define $\theta_{\beta}$ and $\theta_{\alpha}$ by

$$
\theta_{\beta}=\frac{\beta}{2} \sum_{j=1}^{N-1} \frac{1}{\left|1-e^{\mathrm{i} \omega_{j}}\right|^{\beta}}, \quad \theta_{\alpha}=\frac{\alpha}{2} \sum_{j=1}^{N-1} \frac{1}{\left|1-e^{\mathrm{i} \omega_{j}}\right|^{\alpha}} .
$$

where $\mathrm{i}^{2}=-1$. By direct computations, we have following lemma.

Lemma 2.1. For any given $0<\alpha<\beta, N \geq 2, G_{1}(\lambda)$ and $G_{2}(\lambda)$ have following properties.

(i) $G_{1}(\lambda)$ and $G_{2}(\lambda)$ are a monotonically increasing functions in $\lambda$ when $\lambda \geq 2$;

(ii) $\lim _{\lambda \rightarrow \infty} G_{1}(\lambda)=\lim _{\lambda \rightarrow \infty} G_{2}(\lambda)=\infty$; 
(iii) there exists a $\lambda_{1}=\lambda_{1}(N)>0$ such that for any $\lambda \geq \lambda_{1}$,

$$
G_{1}(\lambda) \geq\left(\frac{\theta_{\beta}}{\theta_{\alpha}}\right)^{\frac{1}{\beta-\alpha}}
$$

(iv) there exists a $\lambda_{2}=\lambda_{2}(N)>0$ such that for any $\lambda \geq \lambda_{2}$, we have

$$
G_{2}(\lambda) \geq \max \left\{\left(\frac{\theta_{\beta}}{\theta_{\alpha}}\right)^{\frac{1}{\beta-\alpha}},\left(\frac{\beta}{\alpha}\right)^{\frac{1}{\beta-\alpha}}\right\} .
$$

Then we give the proof of Theorem 1.1.

Proof of Theorem 1.1. To prove $q=\left(Q_{1}, Q_{2}, q_{1}, \ldots, q_{N}\right)$ satisfies (1.6), we calculate $\frac{\partial U}{\partial Q_{j}}$ for $j=1,2$ and $\frac{\partial U}{\partial q_{k}}$ for $1 \leq k \leq N$. For $i, j \in\{1,2\}$, we have

$$
\frac{\partial U}{\partial Q_{j}}=\sum_{k=1}^{N}\left(\frac{\alpha\left(Q_{j}-q_{k}\right)}{\left|Q_{j}-q_{k}\right|^{\alpha+2}}-\frac{\beta\left(Q_{j}-q_{k}\right)}{\left|Q_{j}-q_{k}\right|^{\beta+2}}\right)+\left(\frac{\alpha\left(Q_{j}-Q_{i}\right)}{\left|Q_{j}-Q_{i}\right|^{\alpha+2}}-\frac{\beta\left(Q_{j}-Q_{i}\right)}{\left|Q_{j}-Q_{i}\right|^{\beta+2}}\right) .
$$

Since $Q_{1}=\left(0,0, r_{0}\right), Q_{2}=\left(0,0,-r_{0}\right)$ and $q_{k}=\phi(\lambda) r_{0}\left(\cos \omega_{k}, \sin \omega_{k}, 0\right)$, we have that

$$
\sum_{k=1}^{N}\left(\frac{\alpha}{\left|Q_{j}-q_{k}\right|^{\alpha+2}}-\frac{\beta}{\left|Q_{j}-q_{k}\right|^{\beta+2}}\right)\left(Q_{j}-q_{k}\right)=\left(\frac{N \alpha}{\left|\lambda r_{0}\right|^{\alpha+2}}-\frac{N \beta}{\left|\lambda r_{0}\right|^{\beta+2}}\right) Q_{j},
$$

and

$$
\left(\frac{\alpha}{\left|Q_{j}-Q_{i}\right|^{\alpha+2}}-\frac{\beta}{\left|Q_{j}-Q_{i}\right|^{\beta+2}}\right)\left(Q_{j}-Q_{i}\right)=\left(\frac{2 \alpha}{\left|2 r_{0}\right|^{\alpha+2}}-\frac{2 \beta}{\left|2 r_{0}\right|^{\beta+2}}\right) Q_{j} .
$$

Note that (2.5) and (2.6) yield that for $j=1$ or 2 ,

$$
\frac{\partial U}{\partial Q_{j}}=\left(\frac{N \alpha}{\left|\lambda r_{0}\right|^{\alpha+2}}-\frac{N \beta}{\left|\lambda r_{0}\right|^{\beta+2}}+\frac{2 \alpha}{\left|2 r_{0}\right|^{\alpha+2}}-\frac{2 \beta}{\left|2 r_{0}\right|^{\beta+2}}\right) Q_{j} .
$$

Note that for $j=1,2$,

$$
\operatorname{diag}\left(-\omega_{0}^{2},-\omega_{0}^{2}, 0\right) Q_{j}=0
$$

We have following equality holds.

$$
\frac{\partial U}{\partial Q_{j}}=\frac{N \alpha}{\left|\lambda r_{0}\right|^{\alpha+2}}-\frac{N \beta}{\left|\lambda r_{0}\right|^{\beta+2}}+\frac{2 \alpha}{\left|2 r_{0}\right|^{\alpha+2}}-\frac{2 \beta}{\left|2 r_{0}\right|^{\beta+2}}=0 .
$$

By (2.9), we obtain that

$$
r_{0}=r(\lambda) \equiv \frac{1}{2 \lambda}\left(\frac{\beta\left(2 \lambda^{\beta+2}+2^{2+\beta} N\right)}{\alpha\left(2 \lambda^{\alpha+2}+2^{\alpha+2} N\right)}\right)^{\frac{1}{\beta-\alpha}}
$$

Note that $\lambda>0$ is needed up to now. By (1.8), we have that $r(\lambda)=\frac{G_{1}(\lambda)}{\sqrt{\lambda^{2}-1}}$ when $\lambda \geq 1$. 
Note that $\frac{\partial U}{\partial q_{k}}$ can be calculated by

$$
\frac{\partial U}{\partial q_{k}}=\sum_{j \neq k}\left(\frac{\alpha}{\left|q_{k j}\right|^{\alpha+2}}-\frac{\beta}{\left|q_{k j}\right|^{\beta+2}}\right) q_{k j}+\sum_{j=1}^{2}\left(\frac{\alpha\left(q_{k}-Q_{j}\right)}{\left|q_{k}-Q_{j}\right|^{\alpha+2}}-\frac{\beta\left(q_{k}-Q_{j}\right)}{\left|q_{k}-Q_{j}\right|^{\beta+2}}\right) .
$$

Because following discussion is in $x y$-plane, we identify the $x y$-plane with $\mathbf{C}$. Therefore, $q_{k}=$ $\phi(\lambda) r_{0} e^{\mathrm{i} \omega_{k}}$ and $q_{k j}=\phi(\lambda) r_{0} e^{\mathrm{i} \omega_{k}}\left(1-e^{\mathrm{i} \omega_{j-k}}\right)$. Hence, we have that

$$
\begin{aligned}
\sum_{j \neq k}\left(\frac{\alpha}{\left|q_{k j}\right|^{\alpha+2}}-\frac{\beta}{\left|q_{k j}\right|^{\beta+2}}\right) q_{k j} & =\sum_{j \neq k}\left(\frac{\alpha\left(1-e^{\mathrm{i} \omega_{j-k}}\right) q_{k}}{\left(\phi r_{0}\right)^{\alpha+2}\left|1-e^{\mathrm{i} \omega_{j-k}}\right|^{\alpha+2}}-\frac{\beta\left(1-e^{\mathrm{i} \omega_{j-k}}\right) q_{k}}{\left(\phi r_{0}\right)^{\beta+2}\left|1-e^{\mathrm{i} \omega_{j-k}}\right|^{\beta+2}}\right) \\
& =\sum_{j=1}^{N-1}\left(\frac{\alpha\left(1-e^{\mathrm{i} \omega_{j}}\right) q_{k}}{\left(\phi r_{0}\right)^{\alpha+2}\left|1-e^{\mathrm{i} \omega_{j}}\right|^{\alpha+2}}-\frac{\beta\left(1-e^{\mathrm{i} \omega_{j}}\right) q_{k}}{\left(\phi r_{0}\right)^{\beta+2} \mid 1-e^{\left.\mathrm{i} \omega_{j}\right|^{\beta+2}}}\right) .
\end{aligned}
$$

Note that $\left|1-e^{\mathrm{i} \omega_{j}}\right|=\left|1-e^{\mathrm{i} \omega_{N-j}}\right|=\sqrt{2-2 \cos \omega_{j}}$ and $\left(1-e^{\mathrm{i} \omega_{j}}\right)+\left(1-e^{\mathrm{i} \omega_{N-j}}\right)=2-2 \cos \omega_{j}$. Then we have

$$
\sum_{j=1}^{N-1} \frac{1-e^{\mathrm{i} \omega_{j}}}{\left|1-e^{\mathrm{i} \omega_{j}}\right|^{\alpha+2}}=\frac{1}{2}\left(\sum_{j=1}^{N-1} \frac{1-e^{\mathrm{i} \omega_{j}}}{\left|1-e^{\mathrm{i} \omega_{j}}\right|^{\alpha+2}}+\sum_{j=1}^{N-1} \frac{1-e^{\mathrm{i} \omega_{N-j}}}{\left|1-e^{\mathrm{i} \omega_{N-j}}\right|^{\alpha+2}}\right)=\frac{1}{2}\left(\sum_{j=1}^{N-1} \frac{1}{\left|1-e^{\mathrm{i} \omega_{j}}\right|^{\alpha}}\right) .
$$

Therefore, we can reduce (2.12) to

$$
\begin{aligned}
\sum_{j \neq k}\left(\frac{\alpha}{\left|q_{k j}\right|^{\alpha+2}}-\frac{\beta}{\left|q_{k j}\right|^{\beta+2}}\right) q_{k j} & =\frac{1}{2} \sum_{j=1}^{N-1}\left(\frac{\alpha}{\left(\phi r_{0}\right)^{\alpha+2} \mid 1-e^{\left.\mathrm{i} \omega_{j}\right|^{\alpha}}}-\frac{\beta}{\left(\phi r_{0}\right)^{\beta+2} \mid 1-e^{\left.\mathrm{i} \omega_{j}\right|^{\beta}}}\right) q_{k} \\
& =\left(\frac{\theta_{\alpha}}{\left(\phi r_{0}\right)^{\alpha+2}}-\frac{\theta_{\beta}}{\left(\phi r_{0}\right)^{\beta+2}}\right) q_{k}
\end{aligned}
$$

where $\theta_{\beta}$ and $\theta_{\alpha}$ are given by (2.1). The second summation of (2.11) can be simplified as

$$
\begin{aligned}
\sum_{j=1}^{2}\left(\frac{\alpha\left(q_{k}-Q_{j}\right)}{\left|q_{k}-Q_{j}\right|^{\alpha+2}}-\frac{\beta\left(q_{k}-Q_{j}\right)}{\left|q_{k}-Q_{j}\right|^{\beta+2}}\right) & =\left(\frac{\alpha}{\left(\lambda r_{0}\right)^{\alpha+2}}-\frac{\beta}{\left(\lambda r_{0}\right)^{\beta+2}}\right)\left(q_{k}-Q_{1}+q_{k}-Q_{2}\right) \\
& =\left(\frac{2 \alpha}{\left(\lambda r_{0}\right)^{\alpha+2}}-\frac{2 \beta}{\left(\lambda r_{0}\right)^{\beta+2}}\right) q_{k},
\end{aligned}
$$

Then (2.11) is reduced to

$$
\frac{\partial U}{\partial q_{k}}=\left(\frac{\theta_{\alpha}}{\left(\phi r_{0}\right)^{\alpha+2}}-\frac{\theta_{\beta}}{\left(\phi r_{0}\right)^{\beta+2}}+\frac{2 \alpha}{\left(\lambda r_{0}\right)^{\alpha+2}}-\frac{2 \beta}{\left(\lambda r_{0}\right)^{\beta+2}}\right) q_{k}
$$

$\operatorname{By} \operatorname{diag}\left(-\omega_{0}^{2},-\omega_{0}^{2}, 0\right) q_{k}+\frac{\partial U(q)}{\partial q_{k}}=0$, we have that

$$
\omega_{0}^{2}=\frac{\theta_{\alpha}}{\left(\phi r_{0}\right)^{\alpha+2}}-\frac{\theta_{\beta}}{\left(\phi r_{0}\right)^{\beta+2}}+\frac{2 \alpha}{\left(\lambda r_{0}\right)^{\alpha+2}}-\frac{2 \beta}{\left(\lambda r_{0}\right)^{\beta+2}}
$$


By $\lambda \geq 2$ and (2.10), we have that

$$
\lambda r_{0}=\frac{1}{2}\left(\frac{\beta\left(2 \lambda^{\beta+2}+2^{2+\beta} N\right)}{\alpha\left(2 \lambda^{\alpha+2}+2^{\alpha+2} N\right)}\right)^{\frac{1}{\beta-\alpha}} \geq\left(\frac{\beta}{\alpha}\right)^{\frac{1}{\beta-\alpha}} .
$$

This yields that

$$
\frac{2 \alpha}{\left(\lambda r_{0}\right)^{\alpha+2}}-\frac{2 \beta}{\left(\lambda r_{0}\right)^{\beta+2}} \geq 0
$$

By Lemma 2.1, we have that there exists a $\lambda_{1}=\lambda_{1}(N)$ which depends on $N$ such that for any $\lambda \geq \lambda_{1}, \phi(\lambda) r_{0}=G_{1}(\lambda) \geq\left(\frac{\theta_{\beta}}{\theta_{\alpha}}\right)^{\frac{1}{\beta-\alpha}}$. This yields

$$
\frac{\theta_{\alpha}}{\left(\phi r_{0}\right)^{\alpha+2}}-\frac{\theta_{\beta}}{\left(\phi r_{0}\right)^{\beta+2}} \geq 0
$$

Hence, by (2.18) and (2.19), $\omega_{0}$ satisfying (2.17) is well-defined for any $\lambda \geq \max \left\{2, \lambda_{1}\right\}$.

Then $q(t)=E_{\omega_{0}}(t) q$ is a homographic solution of the system (1.2) where $q$ satisfies (1.5), and $\omega_{0}$ is given by (2.17).

Readers may verify Proposition 1.2 by Lemma 2.1 following the proof of Theorem 1.1. We omit the proof here.

When $N=2$, the solution is a flat non-planar solution. There is a plane $\Pi(t)$ in $\mathbf{R}^{3}$ such that $q(t) \in \Pi(t)$ for all $t \in S_{\tau}$ pair-wisely different. In the celestial mechanics, there does not exist any flat non-planar solution.

Corollary 2.2. (i) (The (2+2)-body problem) When $0<\alpha<\beta, N=2$ and $\lambda \geq 2$, $q(t)$ satisfying (1.5) is the non-planar solution of (1.2). Furthermore, the configuration is always a rhombus.

(ii) (The (3+2)-body problem) When $N=2, \lambda \geq \max \left\{2, \lambda_{2}\right\}, q(t)$ satisfying (1.9) is a nonplanar solution of (1.2). Especially, the configuration of $q(t)$ is always a rhombus where $Q_{3}$ is at the center of this rhombus.

\subsection{One General Periodic Homographic Solution}

In this section, we will prove the Theorem 1.3. Before the proof, we need following lemma.

Lemma 2.3. There exists a $\lambda_{0}$ such that for all $\lambda>\lambda_{0}>0$, following inequality holds.

$$
\begin{aligned}
& \left(N 2^{\beta+2}+2 \lambda^{\beta+2}\right)\left((N-2) 2^{\alpha+2} \phi^{\beta+2}+2 \lambda^{\alpha+2} \phi^{\beta+2}-\theta_{\alpha}(2 \lambda)^{\alpha+2} \phi^{\beta-\alpha}\right) \\
& <\left(N 2^{\alpha+2}+2 \lambda^{\alpha+2}\right)\left((N-2)(2 \phi)^{\beta+2}+2(\lambda \phi)^{\beta+2}-\theta_{\beta}(2 \lambda)^{\beta+2}\right) .
\end{aligned}
$$


Proof. Note that the highest order of $\lambda$ on the both sides of (2.20) are $4 \lambda^{\beta+\alpha+4} \phi^{\beta+2}$. The second highest order of $\lambda$ on the right hand side of (2.20) is $N 2^{\alpha+3}(\lambda \phi)^{\beta+2}$ and the second highest order of $\lambda$ on the left hand side of (2.20) is $(N-2) 2^{\alpha+3} \lambda^{\beta+2} \phi^{\beta+2}-\theta_{\alpha} 2^{\alpha+3} \lambda^{\beta+\alpha+4} \phi^{\beta-\alpha}$. Note

$$
N 2^{\alpha+3}(\lambda \phi)^{\beta+2}>(N-2) 2^{\alpha+3}(\lambda \phi)^{\beta+2}-\theta_{\alpha} 2^{\alpha+3} \lambda^{\beta+\alpha+4} \phi^{\beta-\alpha}
$$

is equivalent to

$$
2^{\alpha+4}(\lambda \phi)^{\beta+2}>-\theta_{\alpha} 2^{\alpha+3} \lambda^{\beta+\alpha+4} \phi^{\beta-\alpha},
$$

It holds because the left hand side is positive and the right hand side is negative when $\lambda>0$. Then we obtain this lemma holds when $\lambda_{0}$ is sufficiently large.

Now, we give the proof of Theorem 1.3.

Proof of Theorem 1.3. We plug $q(t)=r(t) E(t) q_{0}$ defined in (1.12) into the both sides of (1.2) and obtain that the left hand side of (1.2) are

$$
\begin{aligned}
\ddot{Q}_{j}(t) & =(\ddot{r}(t) E(t)+2 \dot{r}(t) \dot{E}(t)+r(t) \ddot{E}(t)) Q_{j}, \\
\ddot{q}_{k}(t) & =(\ddot{r}(t) E(t)+2 \dot{r}(t) \dot{E}(t)+r(t) \ddot{E}(t)) q_{k},
\end{aligned}
$$

where $1 \leq j \leq 2$ and $1 \leq k \leq N$ and on the other hand, by (2.4-2.16), we have

$$
\begin{aligned}
& \frac{\partial U(q)}{\partial Q_{j}}=r(t) E(t)\left(\frac{N \alpha}{(\lambda r)^{\alpha+2}}-\frac{N \beta}{(\lambda r)^{\beta+2}}+\frac{2 \alpha}{(2 r)^{\alpha+2}}-\frac{2 \beta}{(2 r(t))^{\beta+2}}\right) Q_{j}, \\
& \frac{\partial U(q)}{\partial q_{k}}=r(t) E(t)\left(\frac{\theta_{\alpha}}{(\phi r)^{\alpha+2}}-\frac{\theta_{\beta}}{(\phi r)^{\beta+2}}+\frac{2 \alpha}{(\lambda r)^{\alpha+2}}-\frac{2 \beta}{(\lambda r)^{\beta+2}}\right) q_{k} .
\end{aligned}
$$

We define $A(t)$ by

$$
A(t) \equiv r^{-1}(t) E^{-1}(t)(\ddot{r} E(t)+2 \dot{r} \dot{E}(t)+r(t) \ddot{E}(t))=r^{-1}(t)\left(\begin{array}{ccc}
\ddot{r}-r \dot{\omega}^{2} & 0 & 0 \\
0 & \ddot{r}-r \dot{\omega}^{2} & 0 \\
0 & 0 & \ddot{r}
\end{array}\right) .
$$

Then $\ddot{Q}_{j}(t)=r(t) E(t) A(t) Q_{j}=-\frac{\partial U(q(t))}{\partial Q_{j}}$ and (2.25) yield that

$$
\ddot{r}=\frac{N \beta}{\lambda^{\beta+2} r^{\beta+1}}-\frac{N \alpha}{\lambda^{\alpha+2} r^{\alpha+1}}+\frac{2 \beta}{2^{\beta+2} r^{\beta+1}}-\frac{2 \alpha}{2^{\alpha+2} r^{\alpha+1}} .
$$

By $\ddot{q}_{k}=r(t) E(t) A(t) q_{k}=-\frac{\partial U(q(t))}{q_{k}},(2.26)$ and (2.28) yield that

$$
\ddot{r}-r \dot{\omega}^{2}=\frac{\theta_{\beta}}{\phi^{\beta+2} r^{\beta+1}}-\frac{\theta_{\alpha}}{\phi^{\alpha+2} r^{\alpha+1}}+\frac{2 \beta}{\lambda^{\beta+2} r^{\beta+1}}-\frac{2 \alpha}{\lambda^{\alpha+2} r^{\alpha+1}} .
$$


By (2.28) and (2.29), we have that

$$
\dot{\omega}^{2}=\frac{(N-2) \beta}{(\lambda r)^{\beta+2}}-\frac{(N-2) \alpha}{(\lambda r)^{\alpha+2}}+\frac{2 \beta}{(2 r)^{\beta+2}}-\frac{2 \alpha}{(2 r)^{\alpha+2}}-\frac{\theta_{\beta}}{(\phi r)^{\beta+2}}+\frac{\theta_{\alpha}}{(\phi r)^{\alpha+2}} .
$$

If $q(t)$ is the solution of $(1.2), \dot{\omega}^{2} \geq 0$ is a necessary condition, i.e.,

$$
\frac{(N-2) \beta}{(\lambda r)^{\beta+2}}-\frac{(N-2) \alpha}{(\lambda r)^{\alpha+2}}+\frac{2 \beta}{(2 r)^{\beta+2}}-\frac{2 \alpha}{(2 r)^{\alpha+2}}-\frac{\theta_{\beta}}{(\phi r)^{\beta+2}}+\frac{\theta_{\alpha}}{(\phi r)^{\alpha+2}} \geq 0 .
$$

Note that (2.31) is equivalent to for all $t \in \mathbf{R}$,

$$
r \leq \Lambda(\lambda) \equiv \frac{1}{2 \lambda \phi}\left(\frac{\beta\left((N-2)(2 \phi)^{\beta+2}+2(\lambda \phi)^{\beta+2}-\theta_{\beta}(2 \lambda)^{\beta+2}\right)}{\alpha\left((N-2)(2 \phi)^{\alpha+2}+2(\lambda \phi)^{\alpha+2}-\theta_{\alpha}(2 \lambda)^{\alpha+2}\right)}\right)^{\frac{1}{\beta-\alpha}} .
$$

Note that (2.28) can be simplified to

$$
\ddot{r}=-\Psi^{\prime}(r)=\frac{\beta\left(N 2^{\beta+2}+2 \lambda^{\beta+2}\right)}{(2 \lambda r)^{\beta+2}}-\frac{\alpha\left(N 2^{\alpha+2}+2 \lambda^{\alpha+2}\right)}{(2 \lambda r)^{\alpha+2}},
$$

where $\Psi$ is define by (1.13). Note that $\bar{r}$ given by (1.14) is the only positive root of $\Psi^{\prime}(\bar{r})=0$.

As the discussion Theorem 4.1 of [4], there is an $\tau$-periodic oscillating solution of $r(t)>0$ for any given Hamiltonian energy $\Psi(\bar{r})<h<0$. For any solution $r(t)$ which is not the constant solution, i.e., $r(t) \not \equiv \bar{r}$, we must have $t_{1}, t_{2} \in[0, \tau]$ such that $r\left(t_{1}\right) \leq \bar{r}$ and $r\left(t_{2}\right) \geq \bar{r}$

If there exists some $\lambda_{0}$ such that $\Lambda\left(\lambda_{0}\right)>\bar{r}$, then $r(t)$, which is the periodic solution of (2.33), satisfies $\max _{t \in S_{\tau}} r(t)<\Lambda\left(\lambda_{0}\right)$ for the given Hamiltonian energy $h \in\left(\Psi(\bar{r}), \Psi\left(\Lambda\left(\lambda_{0}\right)\right)\right)$. Then for $\lambda_{0}$, $q(t)=r(t) E(\omega(t)) q$ is the solution of the system (1.2).

Next, we prove the existence of $\lambda_{0}$ which yields for any $\lambda \geq \lambda_{0}$, following inequality holds.

$$
\Lambda(\lambda)>\bar{r}
$$

Note that (2.34) holds is equivalent to

$$
\frac{1}{2 \lambda \phi}\left(\frac{\beta\left((N-2)(2 \phi)^{\beta+2}+2(\lambda \phi)^{\beta+2}-\theta_{\beta}(2 \lambda)^{\beta+2}\right)}{\alpha\left((N-2)(2 \phi)^{\alpha+2}+2(\lambda \phi)^{\alpha+2}-\theta_{\alpha}(2 \lambda)^{\alpha+2}\right)}\right)^{\frac{1}{\beta-\alpha}}>\frac{1}{2 \lambda}\left(\frac{\beta\left(N 2^{\beta+2}+2 \lambda^{\beta+2}\right)}{\alpha\left(N 2^{\alpha+2}+2 \lambda^{\alpha+2}\right)}\right)^{\frac{1}{\beta-\alpha}} .
$$

By direct computations, we have that (2.35) holds if and only if (2.20) holds. By lemma 2.3, we have that (2.35) holds.

Therefore, for such $\lambda$ which satisfying (2.34), $q(t)=r(t) E(t) q_{0}$ is a solution of the system where $r(t)$ is the classical solution of the ODE (2.33) and $\omega$ is given by (1.16).

Acknowledgment. This paper is a part of my Ph.D. thesis. I would like to express my sincere thanks to my advisor Professor Yiming Long for his valuable guidance, help, suggestions and encouragements during my study and discussions on this topic. 


\section{References}

[1] M. Corbera, J. Llibre, and E. Pérez-Chavela. Equilibrium points and central configurations for the Lennard-Jones 2- and 3-body problems. Celestial Mech. Dynam. Astronom., 89(3):235-266, 2004.

[2] M. Corbera, J. Llibre, and E. Pérez Chavela. Symmetric planar non-collinear relative equilibria for the Lennard-Jones potential 3-body problem with two equal masses. In Proceedings of the 6th Conference on Celestial Mechanics (Spanish), Monogr. Real Acad. Ci. Exact. Fís.-Quím. Nat. Zaragoza, 25, pages 93-114. Real Acad. Ci. Exact., Fís. Quím. Nat. Zar, Zaragoza, 2004.

[3] R. T. Jones. The n-body problem with repulsive-attractive quasihomogeneous potential functions. $\mathrm{PhD}$ thesis, 2006.

[4] B. Liu, Y. Long, and C. Zeng. Solutions of the generalized Lennard-Jones system. Acta Math. Sin. (Engl. Ser.), 34(2):139-170, 2018.

[5] J. Llibre and Y. Long. Periodic Solutions for the Generalized Anisotropic Lennard-Jones Hamiltonian. Qual. Theory Dyn. Syst., 14(2):291-311, 2015.

[6] L. Sbano and J. Southall. Periodic solutions of the $N$-body problem with Lennard-Jones-type potentials. Dyn. Syst., 25(1):53-73, 2010. 